\title{
Synthesis and Characterization of Silver Nanoparticles Embedded in Silica Matrix
}

José R. Montes Bojórquez ${ }^{1}$, Javier Carrillo Pesqueira ${ }^{1}$, Francisco E. Rojas Gonzalez ${ }^{1}$, Ofelia Hernández Negrete $^{2}$, Hilda E. Esparza Ponce ${ }^{3}$, Roberto Carrillo-Torres ${ }^{1}$, and Javier Hernández Paredes ${ }^{1 *}$

1. Departamento de Física, Universidad de Sonora (UNISON), Edificio 3R, Blvd. Luis Encinas J. y Rosales s/n Col. Centro, Hermosillo, Sonora, C.P. 83000, México

2. Departamento de Ingeniería Química y Metalurgia, Universidad de Sonora (UNISON), Edificio 5I, Blvd. Luis Encinas J. y Rosales s/n Col. Centro, Hermosillo, Sonora, C.P. 83000, México

3. Centro de Investigación en Materiales Avanzados (CIMAV), Miguel de Cervantes 120, Chihuahua, Chihuahua, C.P. 31136, México.

*Corresponding author: jahernandezparedes@gmail.com; javier.hernandez@ unison.mx

In this work, we present the synthesis and characterization of a composite formed by silver nanoparticles (AgNPs) embedded in silica matrix ( $\mathrm{SiO} 2)$. In this regard, there are some works reporting the importance of these systems for optical applications due to the surface plasmon of resonance (SPR) that present the AgNPs [1-3]. There are various preparation methods or techniques for these composites, however, the sol-gel method is a reliable route to obtain this kind of materials [1].

All the chemicals were purchased from Sigma-Aldrich and used as received. For the synthesis of the AgNPs, we have implemented the Lee-Meisel method. $9 \mathrm{mg}$ of AgNO3 ( $\geq 99.9 \%$ pure) were dissolved in $50 \mathrm{~mL}$ of deionized water and heated to boiling. Then a second solution was prepared from $11.5 \mathrm{mg}$ of sodium citrate dissolved in $10 \mathrm{~mL}$ of deionized water and it was added to the first solution. The system was kept on boiling for $1 \mathrm{~h}$ [4]. The solution of AgNPs was analysed using an Ocean Optics USB2000+ UV-VIS-NIR spectrometer. Once the AgNPs were ready, we have proceeded to incorporate them into a silica matrix. The procedure was as follows: $0.2 \mathrm{~mL}$ of tetraethyl orthosilicate (TEOS) ( $\geq$ $99.0 \%$ pure $), 60 \mathrm{~mL}$ of ethanol absolute $(\rho=0.790-0.793 \mathrm{~g} / \mathrm{cm} 3), 20 \mathrm{~mL}$ of deionized water and $100 \mu \mathrm{L}$ of the AgNPs solution were mixed together, then a drop of HNO3 (ACS reagent 68-70\%) as catalyst was added and completely mixed. The solution was placed in a vessel covered with a perforated lid and left to evaporate at room temperature for 1 week. The composites were analysed through a Field Emission Scanning Electron Microscopy FESEM JEOL JSM-7800F, operated at 30 and $15 \mathrm{kV}$, under STEM and backscattering modes.

Figure 1 shows the UV-Vis spectrum of the solution with AgNPs. The spectrum has a broad band centred around $422 \mathrm{~nm}$. This feature corresponds to a wide range of particle sizes, however, the characteristics of this band suggested that most of the AgNPs range between 60-70 nm. Figure 2 shows the STEM images of the AgNPs. It is observed that there are different shapes and broad size distribution of AgNPs, which is in accordance with the characteristics of the UV-Vis spectrum. However, most particle sizes range between $60-70 \mathrm{~nm}$. Figure 3 shows the BSE images of the composite formed by the AgNPs embedded in the silica matrix. The bright spots correspond to regions rich in heavy atoms like Ag. At low magnifications e.g. 1500x, it can be seen a homogeneous distribution of the AgNPs across the matrix, however, it is also observed that the AgNPs form agglomerates. In future works, we will try to control the density and distribution of the AgNPs across the $\mathrm{SiO} 2$ matrix to perform further characterization on the optical properties of these systems.

References: 
[1] M. Epifani, et al., J. Am. Ceram. Soc., 83 (2000), p. 2385.

[2] F. Singh, et al., Nucl. Instr. Meth. B., 311 (2013), p. 5.

[3] O. A. Yeshchenko, et al., Phys. Rev. B., 79 (2009), p. 235438.

[4] P. C. Lee and D. Meisel, J. Phys. Chem. 86 (1982), p. 3391.

[5] The authors acknowledge funding from PRODEP-México through project 511-6/18-8537. J. R. Montes Bojórquez also thank PRODEP-México for a Fellowship. J. Carrillo-Pesqueira and F. RojasGonzález gratefully acknowledge the scholarship provided by CONACyT (México).

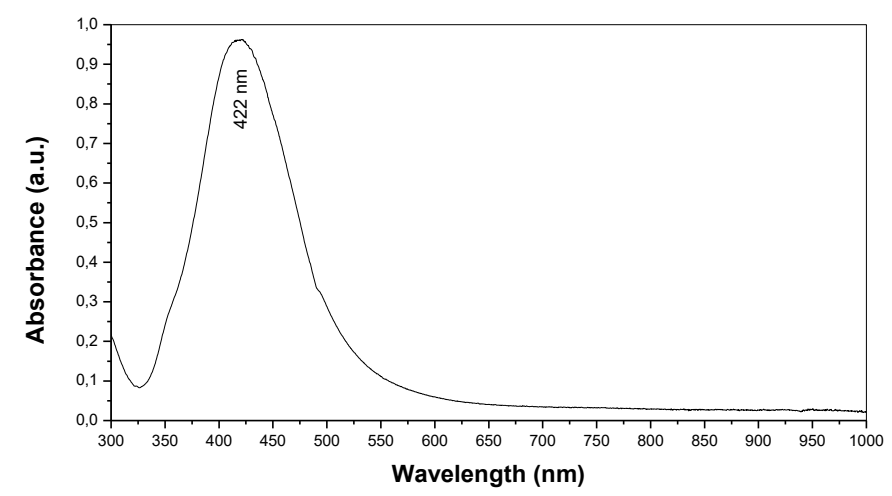

Figure 1. UV-Vis absorption spectrum of the solution with silver nanoparticles (AgNPs). The band is centred at $422 \mathrm{~nm}$, which corresponds to sizes between 60-70 $\mathrm{nm}$.
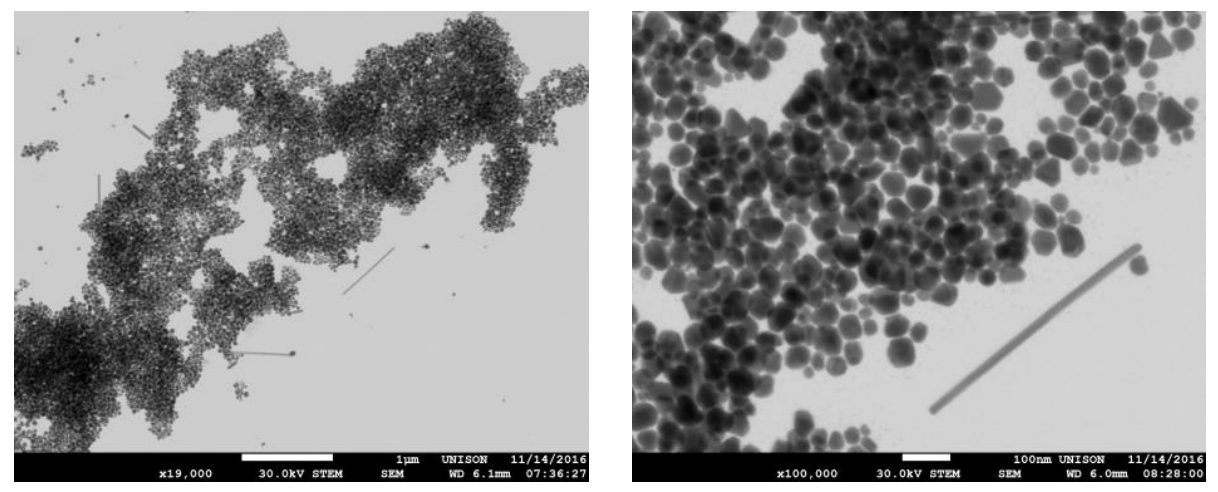

Figure 2. STEM images of the silver nanoparticles (AgNPs) obtained from the Lee-Meisel method at: 19000x left and 100000x right. Scale bars are $1 \mu \mathrm{m}$ and $100 \mathrm{~nm}$, respectively.
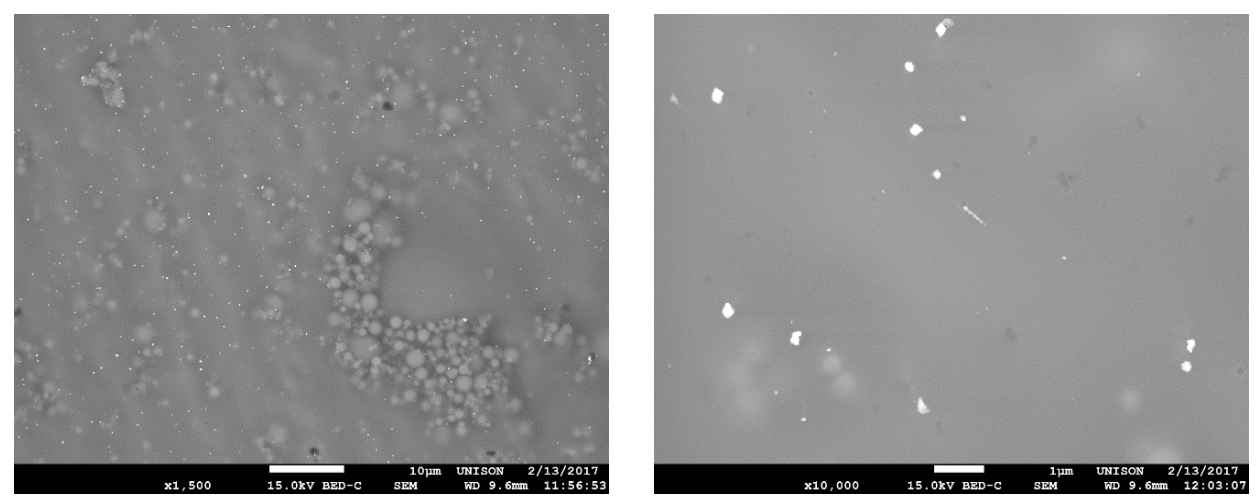

Figure 3. BSE images of the silver nanoparticles embedded in silica matrix at 1500x left and 10000x right. The scale bars are $10 \mu \mathrm{m}$ and $1 \mu \mathrm{m}$, respectively. 